\title{
Novel Visualization of Clostridium difficile Infections in Intensive Care Units
}

\author{
Sean C. Yu ${ }^{1,2}$ Albert M. Lai ${ }^{1} \quad$ Justin Smyer ${ }^{3}$ Jennifer Flaherty ${ }^{3}$ Julie E. Mangino ${ }^{4}$ \\ Ann Scheck McAlearney ${ }^{5,6}$ Po-Yin Yen ${ }^{1}$ Susan Moffatt-Bruce ${ }^{6,7}$ Courtney L. Hebert ${ }^{8}$
}

\footnotetext{
${ }^{1}$ Institute for Informatics, Washington University School of Medicine in St Louis, St Louis, Missouri, United States

2 Department of Biomedical Engineering, Washington University in St Louis, St Louis, Missouri, United States

${ }^{3}$ Department of Clinical Epidemiology, The Ohio State University Wexner Medical Center, Columbus, Ohio, United States

${ }^{4}$ Division of Infectious Diseases, Department of Internal Medicine, The Ohio State University Wexner Medical Center, Columbus, Ohio, United States

${ }^{5}$ Department of Family Medicine, College of Medicine, The Ohio State University, Columbus, Ohio, United States

${ }^{6}$ CATALYST, Center for the Advancement of Team Science, Analytics, and Systems Thinking, College of Medicine, The Ohio State University, Columbus, Ohio, United States

${ }^{7}$ Department of Surgery, College of Medicine, The Ohio State University, Columbus, Ohio, United States

${ }^{8}$ Department of Biomedical Informatics, College of Medicine, Ohio State University, Columbus, Ohio, United States
}

ACI Open 2019;3:e71-e77.
Address for correspondence Courtney L. Hebert, MS, MD, Department of Biomedical Informatics, Ohio State University College of Medicine, Lincoln Tower No. 220S, 1800 Cannon Dr., Columbus, OH 43210, United States (e-mail: Courtney.hebert@osumc.edu).

\section{Abstract}

Keywords

- electronic health records and systems

- biosurveillance and case reporting

- data visualization
Background Accurate and timely surveillance and diagnosis of health care facility onset Clostridium difficile infection ( $\mathrm{HO}-\mathrm{CDI}$ ) is vital to controlling infections within the hospital, but there are limited tools to assist with timely outbreak investigations.

Objectives The objective of this study was to integrate spatiotemporal factors with HO-CDI cases and to develop a map-based dashboard to support infection preventionists (IPs) in performing surveillance and outbreak investigations for HO-CDI.

Methods Clinical laboratory results and Admit-Transfer-Discharge data for admitted patients over 2 years were extracted from the information warehouse of a large academic medical center (AMC) and processed according to the Center for Disease Control National Healthcare Safety Network definitions to classify CDI cases by onset date. Results were validated against the internal infection surveillance database maintained by IPs in Clinical Epidemiology of this AMC. Hospital floor plans were combined with $\mathrm{HO}-\mathrm{CDI}$ case data, to create a dashboard of intensive care units. Usability testing was performed with a think-aloud session and a survey.

Results The simple classification algorithm identified all $265 \mathrm{HO}-\mathrm{CDI}$ cases from January 1 , 2015 to November 30, 2015 with a positive predictive value (PPV) of 96.3\%. When applied to data from 2014, the PPV was 94.6\%. All users "strongly agreed" that the dashboard would be a positive addition to Clinical Epidemiology and would enable them to present hospital-acquired infection information to others more efficiently.

Conclusion The CDI dashboard demonstrates the feasibility of mapping clinical data to hospital patient care units for more efficient surveillance and potential outbreak investigations. received

October 19, 2018

accepted after revision

June 4, 2019

\author{
DOI https://doi.org/ \\ 10.1055/s-0039-1693651. \\ ISSN 2566-9346.
}

(C) 2019 Georg Thieme Verlag KG Stuttgart . New York
License terms

(1) (1) 


\section{Background and Significance}

Clostridium difficile infection (CDI) is a major problem in health care settings, with estimates of the total annual financial burden of health care facility onset-CDI (HO-CDI) ranging from about $\$ 1.5$ billion $^{1}$ to $\$ 3.2$ billion. $^{2}$ In 2011 , Clostridium difficile caused almost 500,000 infections and 29,300 deaths in the United States. ${ }^{3}$ HO-CDI surveillance is mandated by Centers for Medicare and Medicaid services through the Hospital Inpatient Quality Reporting program. ${ }^{4}$ This article discusses the initial development of a tool to help infection preventionists (IPs) more efficiently perform surveillance and tracking of potential outbreaks.

Exposure to $C$. difficile in the hospital setting can come from multiple sources. C. difficile is shed in feces, and contaminated hospital surfaces can serve as a reservoir for spores. ${ }^{5,6} C$. difficile can also be found on the skin and hands of infected patients and health care personnel, often due to contamination from the environment. ${ }^{7}$ Room co-occupancy of patients with CDI has been shown to be associated with development of $\mathrm{CDI}^{8}{ }^{8}$ There is recent evidence that if the prior occupant received an antibiotic, this significantly increases CDI risk for subsequent occupants. ${ }^{9}$ Because of these known risks, IPs within our academic medical center (AMC) keep close track of the numbers, onset times, and locations of CDI cases. At the time of this study, CDI surveillance was performed as follows: IPs in clinical epidemiology received a list of hospitalized patients with laboratory results positive for $\mathrm{CDI}$ and manually determined if the case was an incident, duplicate, or recurrent case. They then determined which cases met the definition for HO- versus community-onset (CO) CDI.

IPs tracked HO-CDI cases in a surveillance database that is distinct from the health system's electronic health records (EHRs). If there is an increase in incidence of CDI, they will investigate any clusters to determine if there is an outbreak, assess for policy breaches, reinforce rigorous compliance with all prevention processes, and hold face-to-face meetings as needed. As part of surveillance, the IPs gather a large amount of information from various sources: (1) case information on HOCDI events from clinical epidemiology databases; (2) information on CO patients from line lists of CDI-positive patients from the laboratory; and (3) where and when the patients were during their stay by chart review in the EHR. Given the considerable time needed to monitor and manage CDI surveillance in this context, we sought to develop an interactive dashboard that included both HO-CDI cases and data about patient movements throughout the hospital.

Tracking the location and movement of patients with CDI within a hospital can be helpful to better understand transmission. ${ }^{10}$ Despite this, integration of indoor spatiotemporal analyses in the EHR for tracking hospital-acquired infection (HAI) is infrequently reported in the literature. A review of spatial and temporal analysis of infection transmission within health care settings ${ }^{10}$ only identified four studies of infection transmission that utilized hospital location. Only one of those studies, by Kho et al, ${ }^{11}$ discussed user-facing visualization tools which were used to track methicillinresistant Staphylococcus aureus (MRSA) and vancomycin- resistant Enterococcus. These tools were used by IPs and nurse managers to educate their nursing staff. In that paper, the authors mention that such dashboards "may be particularly useful in tracking hardy environmental contaminants, such as $C$. difficile, which may concentrate in particular areas/ rooms in a hospital."11 A recent study mapped CDI cases using EHR data; however, representation of hospital units were symbolic (node-based) as opposed to an actual indoor map or blueprint of the hospital. ${ }^{12}$ Another recent study by Cooper et al superimposed the numbers of CDI cases over hospital floor plans but did not subcategorize the CDI cases or perform formal usability testing. ${ }^{13}$

In recent years, the spread of EHR systems have highlighted the importance of usability of software tools in the health care domain for clinicians and other stakeholders. Complicated interfaces that are not consistent with existing workflow lead not only to frustration, but also potentially to medical errors and adverse events. ${ }^{14}$ Any tool, ultimately intended for clinical staff, should be designed with their input, via actual stakeholder usability testing to enhance real-world usability of a system.

The objective of this study was to (1) develop a rule-based algorithm to classify HO-CDI cases from EHR data and (2) design a dashboard that could be used by IPs to perform surveillance and outbreak investigation of HO-CDI. At the time of this study, HO-CDI cases were classified manually, which would not support real-time visualization of cases. The rule-based algorithm was developed to assess the feasibility of operationalizing the dashboard for real-time use. We assessed the algorithm using the IP-curated surveillance database as the gold standard for case classification. We assessed the dashboard with usability testing to optimize the dashboard for relevant users. Though $\mathrm{CDI}$ is present in nonintensive care units (ICUs), we focused on ICU units in this initial design as critically ill patients are at particularly high risk for CDI due to multiple risk factors such as longer length of stay and antibiotic use. ${ }^{15}$

\section{Methods}

\section{Study Setting and Context}

We conducted this study at a large AMC comprising 7 hospitals and over 1,300 beds.

\section{Data}

Laboratory results and Admit-Transfer-Discharge (ADT) data on all patients admitted to the AMC from January 1, 2014 to December 1, 2015 were extracted from the AMC information warehouse and processed in accordance with Center for Disease Control (CDC) National Healthcare Safety Network (NHSN) definitions to classify CDI cases. NHSN tracks HAIs at a national level which is facilitated by standardized definitions. Since the dashboard was designed to be used for routine surveillance, we followed NHSN guidelines as opposed to developing and validating a novel classification model. While identifying CDI cases was simply based on laboratory results, discriminating between types of cases (HO vs. CO) and subtypes of hospital-onset cases (incident, repeat, duplicate, recurrent) required NHSN definitions. 
Ambiguities in the definition, unanticipated exceptions, and difficulties with location data resulted in nontrivial development effort, requiring iteration and validation. Data from 2015 was used to iteratively develop the algorithm and data from 2014 was used to confirm that the algorithm performed well during a separate time period. Data were provided as flat files accessible on a secure internal server, and all processing and classification were done using the $\mathrm{R}$ programming language. The NHSN definition differentiates between incident, recurrent, and repeat cases, and between $\mathrm{HO}$ and $\mathrm{CO}$ cases. ${ }^{16,17}$ According to NHSN, an incident CDI event refers to a $C$. difficile positive laboratory result from a specimen obtained greater than 56 days after the most recent CDI event. If CDI is Present-On-Admission (POA), meaning that the $\mathrm{CDI}$ event occurred on hospital day 1,2 , or 3 , the case is considered CO-CDI. Day 4 onwards, it is considered HO-CDI unless it is considered recurrent, repeat, or duplicate. A recurrent $\mathrm{CDI}$ event refers to a $C$. difficile positive laboratory result from a specimen obtained greater than 14 days and less than or equal to 56 days after the most recent CDI event. A repeat $C D I$ event refers to a $C$. difficile positive laboratory result from a specimen obtained $\leq 14$ days ( 2 weeks) after the most recent CDI event and may also be classified as a duplicate if within that window. Duplicate CDI events are a subset of repeat CDI events where the locations of the related events are the same.

Unit/location of attribution was determined by applying the rule that the location of attribution is the unit/location in which the patient was housed the day before the date of event. If there is more than one unit/location, then the first unit/ location the patient was in on the day before the date of the laboratory event is used. This accounts for the possibility that the patient was transferred to a new location on the date of event, in which case, according to the Transfer Rule, ${ }^{17}$ the unit of attribution is the transferring/discharging location. The unit/location of attribution is only used for internal reporting purposes for $\mathrm{HO}-\mathrm{CDI}$; all external reporting is done according to the Laboratory-Identified Event reporting guidelines per NHSN/CDC. IPs were consulted to clarify or correct misinterpretations of the NHSN guidelines. On occasion, chart review was performed to understand and resolve discrepancies between our results and the IP-curated surveillance database. To verify the validity of our simple rule-based algorithm, we calculated the positive predictive value (PPV) and sensitivity for the identification of HO-CDI cases (as opposed to CO-CDI or non-CDI cases). The IP-curated surveillance database served as the gold standard for case classification.

\section{Floor Plan Data}

Hospital floor plan data for this AMC was extracted from a database provided by the university's information technology (IT) services. The PDF floor plan for select ICUs were manually annotated and then used to construct the visualization tool. The overall data processing strategy can be seen in - Fig. 1 .

\section{HAI Dashboard}

The visualization tool includes an indoor map of the AMC ICUs with user interface elements overlaid on top to repre-

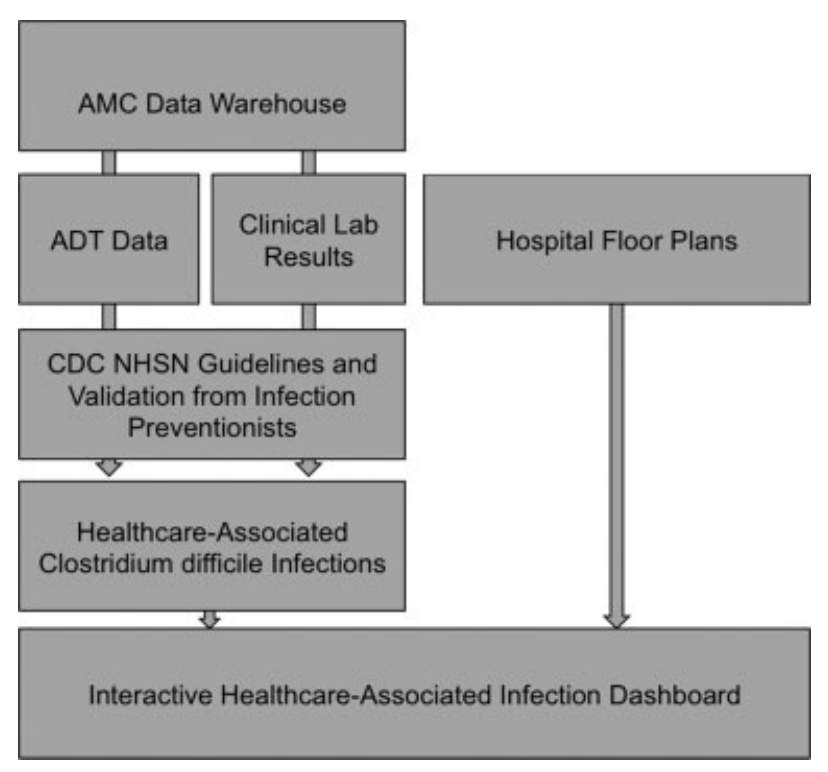

Fig. 1 Data processing strategy.

sent specifics of the HO-CDI cases (-Fig. 2). The numeric label and the size of the dots in the centers of rooms represent the number of HO-CDI events within those rooms as the location of attribution within the adjustable time range. The rooms are also shaded in red proportionate to the number of days the HO-CDI patients were housed in that room from 2 days before to 7 days after the date of event (e.g., presumptive time frame whereby the patient with CDI would most likely be contaminating their environment). As such, the shading represents the approximate risk of contamination/shedding in rooms during the designated time. The user can select single or multiple rooms which will populate the room-based drilldown table that lists all patients who were housed in the room during the chosen time periods. Clicking on individual patients in the room-based drilldown table populates the patient-based drilldown table that shows information about a particular patient including all the rooms that the patient was in, the time of entry and exit from these rooms (ADT information), date of CDI onset, and unit/location of attribution. The dashboard was displayed using Tableau Desktop (Tableau Software, Seattle, Washington, United States).

\section{Usability Testing}

We performed a formal usability study including a thinkaloud protocol ${ }^{18}$ and an anonymous survey on each prototype of the visualization tool with four clinical epidemiology staff (i.e., IPs and clinicians). We recruited clinical epidemiology staff as they have a high level of domain knowledge and because they are the intended end users for tools related to HAI surveillance. The think-aloud protocol allows for freeform feedback that could include feelings, nuanced discussions, and suggestions for the tool, whereas the survey allows for more structured, quantifiable feedback.

Prior to introducing the think-aloud protocol, we provided a quick primer as to the nature and purpose of the tool. We then encouraged users to speak out loud while exploring the 


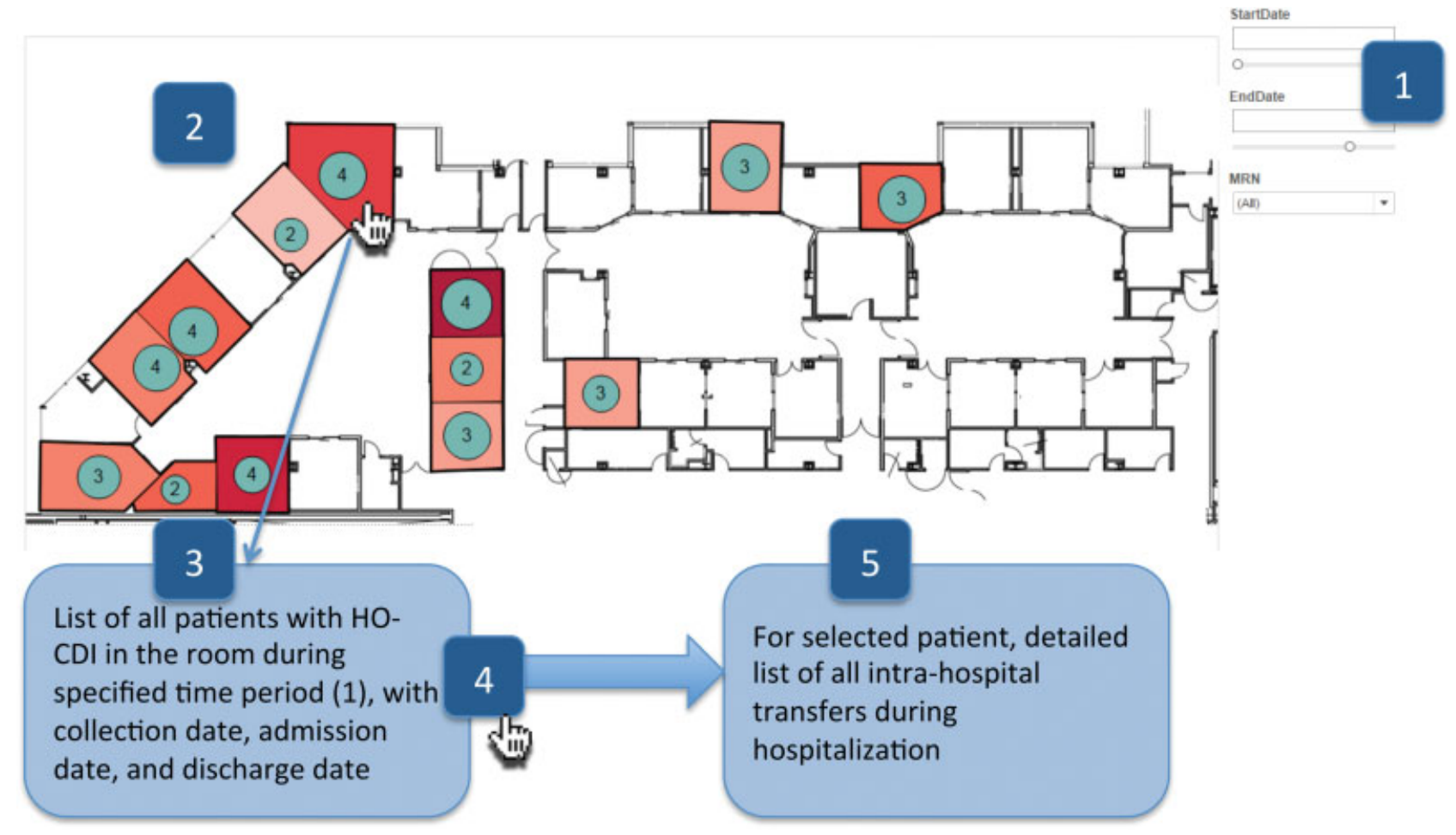

Fig. 2 Functionality of health care facility onset Clostridium difficile infection (HO-CDI) dashboard. (1) User chooses a start and end date. All HOCDI in the visualized unit during that time period are displayed on the map. (2) User selects a room. (3) Table of all patients with HO-CDI in room appears at bottom left. (4) User chooses an individual patients. (5) Table of all intrahospital transfers for the selected patients.

dashboard on their own as well as while performing specific tasks such as adjusting the time range, accessing ADT information of specific patients, and identifying potential outbreak clusters.

After conducting the think-aloud protocol, we had the users fill out a survey assessing the usability of the dashboard based on the Health Information Technology Usability Evaluation Scale (Health-ITUES). ${ }^{19}$ Health-ITUES provides the framework for developing a structured survey by outlining a specific set of critical categories to be evaluated such as perceived usefulness and perceived ease of use. We applied the framework to develop survey questions specific to our HO-CDI dashboard.

All aspects of this study were approved by the AMC's institutional review board.

\section{Results}

\section{HO-CDI Algorithm}

There were 636 positive laboratory results for CDI throughout our multihospital health system between January 1 , 2015 to November 30, 2015. These laboratory results are associated with 626 unique patient encounters, of which 43 were recurrent cases and 10 were repeat cases. Of the remaining 583 cases, 310 were classified as POA, thus representing the CO-CDI classification, leaving 273 as HOCDI cases. Ten of these 273 cases were not found in the IPs' database (gold standard), and were considered false positives, which resulted in a PPV of $96.3 \%$. A subsequent chart review, however, revealed that 2 of 10 were indeed HO-CDI making the true PPV at $97.1 \%$. The gold standard database included 265 cases that were all identified by the algorithm, resulting in zero false negatives, and sensitivity of $100 \%$. When the algorithm was applied to the 2014 data, the PPV was $94.7 \%$ and sensitivity was $92.8 \%$ compared with the gold standard.

\section{Dashboard}

HO-CDI cases were visualized on the dashboard for select ICUs. - Fig. 2 shows the functionality of the HO-CDI dashboard displaying one of these units along with fabricated HOCDI cases.

\section{Usability Testing}

Results of usability testing of the dashboard are outlined in - Table 1. The responses were based on the Likert scale where 1 corresponds to strongly disagree and 5 corresponds to strongly agree. All users $(n=4)$ "strongly agreed" that the dashboard would be a positive addition for clinical epidemiology and would allow them to present HAI information to others more effectively. All "agreed" or "strongly agreed" that they felt confident in manipulating the dashboard to demonstrate it to others, and that the dashboard was easy to learn and use. Comments during the think-aloud sessions were centered on specific features such as improving the intuitiveness of changing the date ranges, adding enteric contact isolation information, and showing $\mathrm{CO}-\mathrm{CDI}$ cases in addition to HO-CDI. Interestingly, users commented that the dashboard might also help assist in the room cleaning process via environmental services staff notifications, by 
Table 1 Results of the usability survey given to infection preventionists $(n=4)$ with possible Likert scale responses ranging from strongly disagree (1) to strongly agree (5)

\begin{tabular}{|l|l|}
\hline Questions & $\begin{array}{l}\text { Median response } \\
\text { on the Likert scale }\end{array}$ \\
\hline $\begin{array}{l}\text { The HAI dashboard will be a positive addition to the department of Clinical Epidemiology at } \\
\text { OSUMC, and for OSUMC at large }\end{array}$ & 5 \\
\hline $\begin{array}{l}\text { The HAI dashboard will help me identify HAI cases and outbreaks more efficiently compared to } \\
\text { manual chart review }\end{array}$ & 5 \\
\hline $\begin{array}{l}\text { The HAI dashboard will help me recommend downstream actions more effectively compared to } \\
\text { manual chart review }\end{array}$ & 5 \\
\hline $\begin{array}{l}\text { The HAI dashboard will help me report cases to CDC's NHSN in a timelier manner compared to } \\
\text { manual chart review }\end{array}$ & 2.5 \\
\hline The HAI dashboard will help me present HAI information to others more effectively & 5 \\
\hline The HAI dashboard contained sufficient and necessary information for me to take further action & 4.5 \\
\hline $\begin{array}{l}\text { The HAI dashboard contained an excess of unnecessary information, which hindered my ability to } \\
\text { use it effectively }\end{array}$ & 2.5 \\
\hline I feel I am confident to manipulate HAI dashboard so that I can demonstrate it to other people & 4 \\
\hline I found it easy to learn how to use HAI dashboard & 5 \\
\hline $\begin{array}{l}\text { The HAI dashboard will help me accomplish my duties within Clinical Epidemiology in a timelier } \\
\text { manner compared to the current workflow of manual chart review }\end{array}$ & 5 \\
\hline \begin{tabular}{l} 
In the future, I will use the HAI dashboard as the primary method of HAl surveillance \\
\hline
\end{tabular} & 4 \\
\hline
\end{tabular}

Abbreviations: CDC, Center for Disease Control; HAI, hospital-acquired infection; NHSN, National Healthcare Safety Network; OSUMC, Ohio State University Wexner Medical Center.

prioritizing rooms to be terminally cleaned and treated with ultraviolet-continuous disinfection, employed for all known rooms with patients infected with CDI. Users also mentioned that the dashboard could assist with communication not only among those in clinical epidemiology, but also with specific units and hospital administration/leadership closer to real time.

\section{Discussion}

\section{Summary}

In this study, we developed an interactive map-based dashboard for the purposes of improving HO-CDI surveillance and outbreak investigation. HO-CDI identification was automated, based on CDC NHSN definitions, using laboratory and ADT data from the AMC information warehouse. All 265 HO-CDI cases between January 1, 2015 to November 30, 2015 were identified by our simple algorithm with a PPV of 96.3\%. Without any adjustments, the algorithm also identified the vast majority of incident HO-CDI cases from 2014 (PPV of 94.6\%). Results of usability testing were positive overall with potential end users strongly agreeing that the dashboard would be a positive addition for clinical epidemiology. The lower score for "The HAI dashboard will help me report cases to CDC's NHSN in a timelier manner compared to manual chart review" is likely because not all interviewed participants perform CDI manual review specifically, but all are involved in cluster/outbreak investigation. In hindsight, better wording may have allowed users who were not specifically performing CDI surveillance to answer more meaningfully. The statement, "The HAI dash- board contained an excess of unnecessary information, which hindered my ability to use it effectively" received a middling score. The version of the dashboard presented to the users included numerous extraneous columns such as if/when the patient was previously admitted, if that previous admission was a CDI case, the admitting service for each admission, calculated length of stay for each admission, and so on. Though these data elements may be useful for individual case investigation, they were deemed unnecessary for the purposes of this tool. A deeper dive into individual cases would involve the EHR where more granular details can be found.

Our study primarily focused on feasibility, utility, and usability of displaying CDI cases on a dashboard among maps of ICUs in multiple hospitals. We believe that there is room for extending our dashboard to investigate other infectious diseases, statistical cluster detection, incorporating strain typing, tracking provider movement, and even providing decision support for patient placement and/or room cleaning.

\section{Next Steps}

In this AMC, we have transitioned this work to hospital IT to develop a near-real-time, operationalized process for automation of CDI detection among all of the patient care units in all hospitals. We are working on an operational version of the dashboard based on lessons learned from this initial feasibility assessment. However, there are various issues to consider when bringing the dashboard to operation. First, new features based on user feedback must also be added and tested, including but not limited to: adding non-HO-CDI 
cases to the dashboard, adding visualizations for patients under isolation protocol, and visualizations for patient movement. Second, we are still deciding what the appropriate platform for this operational dashboard will be. The proposed architecture must be compatible with the existing IT and facilities infrastructure. Third, ownership of the project will need to be transferred cleanly from the research enterprise to the institution's IT department and room renovations on patient care units need to be incorporated/ updated on blueprints. Translation of informatics tools is often a challenge and if surveillance definitions change, there needs to be a process to make these changes quickly and easily. Finally, there must be stringent security rules in place to limit access to those who require it within their operational or clinical roles. Our vision is to have the CDI dashboard eventually embedded in the EHR for relevant users as has been done with other visualization tools. ${ }^{20}$

We also plan to continue to work on automatically identifying clusters of cases, and visualizing them on the dashboard, to augment clinical epidemiology's ability to identify outbreaks closer to real time. Other patient conditions including HAIs, such as MRSA bacteremia, carbapenemresistant Enterobacteriaceae, and even occurrences of patient falls, may also be better understood by considering the information contributed by hospital patient care unit maps. In future work, we anticipate additional applicability to other HAIs to further leverage our surveillance approach to include spatiotemporal visualization of potential clusters. We believe this work will provide important, new information to infection prevention to make the EHR work for us in surveillance, reporting, and control of HAIs.

\section{Limitations}

There are several limitations to our study. The data source for our study was EHR and ADT data from the AMC's information warehouse and is thus susceptible to the usual challenges such as incomplete or erroneous documentation and extraction. While usability testing was appropriately performed, we were only able to test the dashboard on a small number of users, and their perspectives may not represent the sentiment of all potential users. However, given the nature of these discrete laboratory-identified data, we were limited in the number of users with relevant subject matter expertise in this area, at our institution, who were not already involved in development of this application.

\section{Conclusion}

By integrating available data from our EHR, we were able to develop an automated HO-CDI identification algorithm based on the NHSN guidelines for surveillance and display the results on an interactive map of this AMC's ICUs. Responses from the initial round of usability testing of this dashboard were positive. Expanding the concept of HAI surveillance by including spatiotemporal information may have an important impact on our ability to better manage and prevent HAIs and other incidents related to the hospital environment in the future.

\section{Clinical Relevance Statement}

Automation of CDI surveillance allows for less time spent performing manual chart review and allows IPs to focus on advanced epidemiological tasks. Clinical staff expressed overall satisfaction with the current iteration and future direction of the CDI dashboard.

\section{Protection of Human and Animal Subjects}

All research activities reported in this publication were reviewed and approved by the AMC's institutional review board.

\section{Funding}

This project was supported by the Institute for the Design of Environments Aligned for Patient Safety (IDEA4PS) at The Ohio State University which is sponsored by the Agency for Healthcare Research \& Quality (AHRQ) (P30HS024379). The opinions expressed in this document are those of the authors and do not reflect the official position of AHRQ.

\section{Conflict of Interest}

None declared.

\section{References}

1 Zimlichman E, Henderson D, Tamir O, et al. Health care-associated infections: a meta-analysis of costs and financial impact on the US health care system. JAMA Intern Med 2013;173(22):2039-2046

2 O'Brien JA, Lahue BJ, Caro JJ, Davidson DM. The emerging infectious challenge of Clostridium difficile-associated disease in Massachusetts hospitals: clinical and economic consequences. Infect Control Hosp Epidemiol 2007;28(11):1219-1227

3 Lessa FC, Mu Y, Bamberg WM, et al. Burden of Clostridium difficile infection in the United States. N Engl J Med 2015;372(09):825-834

4 Quality Net. Measures. Available at: https://www.qualitynet.org/ dcs/ContentServer?c=Page\&pagename $=$ QnetPublic\%2FPage\% 2FQnetTier3\&cid=1138900298473. Accessed June 26, 2019

5 Faires MC, Pearl DL, Berke O, Reid-Smith RJ, Weese JS. The identification and epidemiology of methicillin-resistant Staphylococcus aureus and Clostridium difficile in patient rooms and the ward environment. BMC Infect Dis 2013;13:342

6 Barra-Carrasco J, Paredes-Sabja D. Clostridium difficile spores: a major threat to the hospital environment. Future Microbiol 2014; 9(04):475-486

7 Weber DJ, Anderson DJ, Sexton DJ, Rutala WA. Role of the environment in the transmission of Clostridium difficile in health care facilities. Am J Infect Control 2013;41(5, Suppl):S105-S110

8 Echaiz JF, Veras L, Zervos M, Dubberke E, Johnson L. Hospital roommates and development of health care-onset Clostridium difficile infection. Am J Infect Control 2014;42(10):1109-1111

9 Freedberg DE, Salmasian H, Cohen B, Abrams JA, Larson EL. Receipt of antibiotics in hospitalized patients and risk for clostridium difficile infection in subsequent patients who occupy the same bed. JAMA Intern Med 2016;176(12):1801-1808

10 Davis GS, Sevdalis N, Drumright LN. Spatial and temporal analyses to investigate infectious disease transmission within healthcare settings. J Hosp Infect 2014;86(04):227-243

11 Kho A, Johnston K, Wilson J, Wilson SJ. Implementing an animated geographic information system to investigate factors associated with nosocomial infections: a novel approach. Am J Infect Control 2006;34(09):578-582

12 Murray SG, Yim JWL, Croci R, et al. Using spatial and temporal mapping to identify nosocomial disease transmission 
of Clostridium difficile. JAMA Intern Med 2017;177(12):18631865

13 Cooper PH, Haney NM, Morey JM. Visual Analysis of Infection Surveillance. Poster Presented at the 2014 Workshop on Visual Analytics in Healthcare, American Medical Informatics Association 2014. Washington, DC; 2014

14 Middleton B, Bloomrosen M, Dente MA, et al; American Medical Informatics Association. Enhancing patient safety and quality of care by improving the usability of electronic health record systems: recommendations from AMIA. J Am Med Inform Assoc 2013;20 (e1):e2-e8

15 Riddle DJ, Dubberke ER. Clostridium difficile infection in the intensive care unit. Infect Dis Clin North Am 2009;23(03):727-743

16 CDC NHSN. Multidrug-Resistant Organism \&amp; Clostridium difficile Infection (MDRO/CDI) Module; 2017. Available at: https://www.cdc.gov/nhsn/pdfs/pscmanual/12pscmdro_cdadcurrent.pdf. Accessed June 26, 2019

17 CDC NHSN. Identifying Healthcare-associated Infections (HAI) for NHSN Surveillance; 2017 [November 21, 2017]. Available at: https://www.cdc.gov/nhsn/PDFs/pscManual/2PSC_IdentifyingHAIs_ NHSNcurrent.pdf. Accessed June 26, 2019

18 Lewis CH. Using the "Thinking Aloud" Method in Cognitive Interface Design (Technical Report) 1982. IBM. RC-9265

19 Yen PY, Wantland D, Bakken S. Development of a customizable health IT usability evaluation scale. AMIA Annu Symp Proc 2010; 2010:917-921

20 Foraker RE, Shoben AB, Lopetegui MA, et al. Assessment of Life's Simple 7 in the primary care setting: the Stroke Prevention in Healthcare Delivery EnviRonmEnts (SPHERE) study. Contemp Clin Trials 2014;38(02):182-189 